\title{
RELATION BETWEEN MOTILITY, ACCELERATED AGING AND GENE EXPRESSION IN SELECTED DROSOPHILA STRAINS UNDER HYPERGRAVITY CONDITIONS
}

\author{
Paloma Serrano ${ }^{1}$, Jack J.W.A. van Loon ${ }^{1,2}$, F. Javier Medina ${ }^{3}$, Raúl Herranz ${ }^{1,3 *}$ \\ ${ }^{1}$ European Space Research \& Technology Center - European Space Agency (ESTEC-ESA), NETHERLANDS; \\ paloma.serrano@awi.de \\ ${ }^{2}$ Dutch Experiment Support Center (DESC) @ OCB-ACTA, VU-University Amsterdam, NETHERLANDS; \\ J.vanLoon@,vumc.nl \\ ${ }^{3}$ Centro de Investigaciones Biologicas (CSIC), C/ Ramiro de Maeztu,7 CP 28040 Madrid, SPAIN;fjmedina@cib.csic.es; \\ *Corresponding author: rherranz@,cib.csic.es, phone: +34 918373112 \#4260, fax: +34 915360432
}

\begin{abstract}
Motility and aging in Drosophila have proven to be highly modified under altered gravity conditions (both in space and ground simulation facilities). In order to find out how closely connected they are, five strains with altered geotactic response or survival rates were selected and exposed to an altered gravity environment of $2 g$. By analysing the different motile and behavioural patterns and the median survival rates, we show that altered gravity leads to changes in motility, which will have a negative impact on the flies' survival. Previous results show a differential gene expression between sessile samples and adults and confirm that environmentally-conditioned behavioural patterns constrain flies' gene expression and life span. Therefore, hypergravity is considered an environmental stress factor and strains that do not respond to this new environment experience an increment in motility, which is the major cause for the observed increased mortality also under microgravity conditions. The neutral-geotaxis selected strain (strain M) showed the most severe phenotype, unable to respond to variations in the gravitational field. Alternatively, the opposite phenotype was observed in positive-geotaxis and long-life selected flies (strains B and L, respectively), suggesting that these populations are less sensitive to alterations in the gravitational load. We conclude that the behavioural response has a greater contribution to aging than the modified energy consumption in altered gravity environments.
\end{abstract}

Keywords: Drosophila; motility; aging; altered gravity.

RUNNING TITLE: Fly Mortality and Aging in Altered Gravity 


\section{INTRODUCTION}

All living organisms are well adapted to the different environmental conditions on Earth, including the gravity. The exposure of adapted organisms to an alien gravity environment could be speculated to result in behavioural and life span changes (as a consequence of short-term alterations in the gravity vector) and perhaps in evolutionary changes (for long term exposures). Previous experiments in space (unmanned satellites, space shuttle or the International Space Station, ISS) have shown that adult Drosophila flies experience a change in their motile behaviour in microgravity conditions (Benguria et al. 1996; Marco et al. 1992; Marco et al. 1986). These experiments show a consistent increase in motility in recently hatched flies whereas mature flies (two week-old individuals) present a slightly increased motility in space (de Juan et al. 2007).

In microgravity research, Drosophila melanogaster flies are considered a model organism for physiological experiments on living organisms. Drosophila reproduce rapidly, have short life cycles, are inexpensive and easy to handle, and can be genetically manipulated at the molecular level. These features allow the accurate design of experiments, as well as new fly generations every month and thus, the possibility to monitor the evolution of a certain parameter/gene expression over generations. In addition, the Drosophila genome and physiology are studied in detail. Around $75 \%$ of the known disease genes in humans have a match in the genome of the fruit fly (Reiter et al. 2001), and $50 \%$ of fly protein sequences have mammalian paralogs. As a result, Drosophila is being used as a genetic model to study mechanisms underlying diabetes, cancer or oxidative stress and aging, the latter aspect being the focus of this study, both on Earth (Lim et al. 2006) and in Space (Benguria et al. 1996).

In addition to space flight, ground based facilities are used to create altered-gravity conditions (Herranz et al. 2012a). Simulated microgravity environments can be recreated on a random positioning machine (RPM) by clinorotation (van Loon 2007) and hypergravity habitats by centrifugation. Magnetic levitation can simulate both environments simultaneously, offering a constant compensation of gravity versus the effects of the RPM/clinorotation that compensate gravity by randomization of the $g$ vector with time (Beaugnon, Tournier 1991a, b; Valles, Guevorkian 2002). As a consequence, modified behavioural patterns that quickly respond to altered gravity conditions will be better studied by simulation with a magnetic field. It was also proven that the locomotor activity of flies is enhanced in microgravity conditions (both on the RPM or under effective force $0 g^{*}$ into a high magnetic field of around 10Tesla, as indicated by the $*$ ) and repressed in hypergravity (both $2 g$ in a centrifuge and $2 g^{*}$ with the magnet), which is in agreement with previous space experiments. Nevertheless, both altered environments have a negative impact in the overall survival of the adult flies (Herranz et al. 2008; Hill et al. 2012).

Moreover, global gene expression profiles in altered gravity from full-genome cDNA microarrays have been obtained in different ground based facilities and in real microgravity (ISS). The comparison of the ISS and RPM samples produce a similar global expression profile only when suboptimal environmental conditions (observed during the space experiment) are applied (Herranz et al. 2010). On ground, using both magnetic or mechanical simulation facilities (Herranz et al. 2012b), we observed different global expression profiles depending on the environmental conditions and the population content (gender, developmental stage, age...).

An open question in Drosophila space research in the last thirty years is the relation between the natural behaviour of the flies, negative geotaxis on Earth (the gravitational signal on Earth allows the flies to look for food or escape from predators) and the accelerated aging in space, also found in hypergravity environments. In other words, is the reduced survival found in altered-gravity conditions related to the motile patterns that the flies present in such environments?

The aim of this paper is to analyse the relationship between motile behaviour and accelerated aging in Drosophila flies by exposing six selected strains to altered-gravity conditions, namely to twice Earth's gravity to support previous results obtained in the opposite environment: simulated microgravity conditions. We aim to prove that the observed changes in motility (increased in microgravity/ reduced in hypergravity) cause a speed-up in aging and therefore, a reduction of the life span or otherwise, survival and locomotor activity are independent parameters.

\section{MATERIALS AND METHODS}

\section{Selection of Drosophila altered behaviour and aging strains}

Previous to the experiments, five Drosophila strains were selected over at least thirty generations. The selection was independently based on two parameters: 1) Geotactic behaviour and 2) Survival.

The altered geotactic behaviour strains (strains A, M and $\mathrm{B}$ ) have been selected for 30 generations and reselected just before the experiment over ten additional generations according to their geotactic 
response in a vertically-oriented ten-exit maze. This maze is opaque and light intensity is kept similar in all the exits, not to interfere with the selection process. Tubes containing food were placed at all exits as enhancers to find the way out. Once the selective process was completed, the behaviour of the flies at $1 g$ resembles an exaggerated but natural escape response from ground (negative geotaxis, strain A), a neutral behaviour to gravity (strain M) and an opposite behaviour to gravity (positive geotaxis, strain B).

The altered-aging strains have been selected for over two decades according to the longevity of their parents to experimentally produce short and a longlife offspring (strains $\mathrm{S}$ and $\mathrm{L}$, respectively). To achieve this goal, eggs from $<1$ week-old imagoes (strain S) and from 4 to 6 week-old imagoes (strain L) were taken to give rise to the following generation of short and long-life flies, respectively. The differential survival between these strains was experimentally tested (median survival is $60 \%$ greater in the strain L, (Serrano et al. 2010)). Note that the Drosophila wild-type (Wt) laboratory population has a natural phenotype similar to A (negative geotaxis, escape response from ground) and $\mathrm{S}$ (short life cycle) but slightly milder.

\section{Experimental design at Ground Based Altered Gravity Facilities}

The Large Diameter Centrifuge (LDC) is located at ESA-ESTEC (Noordwijk, The Netherlands) and belongs to the TEC-MMG section. The LDC is an eight-meter diameter centrifuge able to spin up to $20 \mathrm{~g}$. It consists of six gondolas equipped with technology to perform experiments of very different natures (from a microscope, to cameras, temperature sensors etc) and follow them up in real time (van Loon et al. 2008). Samples placed into a reference gondola in the rotation centre have been used as $1 \mathrm{~g}$ controls.

1) Motility. 25 males and 25 females belonging to each of the six strains were videotaped during two hours at $2 g$ in the $\mathrm{LDC}$ at $20^{\circ} \mathrm{C}$. In the case of the geotactic strains (A, B \& $\mathrm{M})$, recently hatched imagoes (one to two days after hatching) were used for the experiment. On the other hand, recently hatched and two-week old imagoes of the survival strains (S \& L) and wild-type imagoes of the same age were exposed to the mentioned conditions. Motile behaviour was evaluated by direct observation during the first two minutes of the experiment (initial response), then after 30 minutes (initial adaptation) and again after 90 minutes (late adaptation).

2) Survival. Recently hatched imagoes (one to two days old, 50 males and 50 females) of the five selected strains (altered geotaxis, A, M \& B, or survival, $\mathrm{S} \& \mathrm{~L})$ and wild-type Oregon R Drosophila (Wt) were spun at $2 g$ in the LDC for 10 days at $20^{\circ} \mathrm{C}$. LED lights were placed next to the tubes to provide a more natural environment and minimize the impact that complete darkness could have on their behaviour. Survival after the exposure experiments was evaluated during a 50-day followup phase consisting of counting the remaining living individuals in order to study the effects of altered gravity on the survival of the different strains. Survival curves, based on the last observation carried forward (LOCF), were drawn using the Kaplan-Meier method (Motulsky 1995). Two methods were used to test the survival results. Firstly, the R-package statistical software (R_Core_Team 2011) was used to run a log rank test, which is a nonparametric hypothesis test used to compare the survival distribution of the experimental and the control groups of the different strains. Secondly, the 95\% Confidence Intervals (CI) of fractional survival (p) when the median is reached was estimated where $\mathrm{CI}=\mathrm{p} \pm 1,96 \sqrt{ }((1-\mathrm{p}) / \mathrm{N})$ (Altman 1991).

\section{RESULTS}

\section{1) Motility \& Behaviour}

We observed an increase in certain behavioural patterns (leg and wing movements) in the flies exposed to hypergravity during the first two minutes indicating that the change in gravitational load is detected. However, motility remained rather low in all samples during the three observation windows (less than a $5 \%$ of the flies moved, and all displacements were short walks). Only the $\mathrm{M}$ strain (neutral responders to gravity) showed an increased motility at $2 g$ compared to the $1 g$ control, although the number of flies moving was too low to obtain statistically meaningful quantitative results. The observations from the adaptation period, showed less movements in the LDC samples than in the $1 \mathrm{~g}$ controls, confirming previous results of lower activity at $2 g$ in the magnet (Hill et al. 2012). No meaningful differences between males and females were detected.

\section{2) Aging \& Survival}

The survival after a ten-day exposure to hypergravity conditions $(2 g)$ was analysed for each strain by comparing them to a control group of the corresponding strain kept at $1 \mathrm{~g}$. Results show that the three strains with "natural" behaviour (Wt, S, short life and A, negative-geotaxis response strains) show similar survival curves in hypergravity and in control conditions, although some disturbances appear during the first days in the wild type strain. On the contrary, clear differences in survival rates appear for strains $\mathrm{M}, \mathrm{B}$ and $\mathrm{L}$ when compared to 
their respective controls. The strain $M$ (neutralgeotaxis response) presents the most affected survival curve, showing a significant decrease on the survival of the individuals undergoing hypergravity conditions ( $p=7.62 \mathrm{e}-07)$. In contrast, the survival of the strains B (positive-geotaxis responders) and L (long-life) is enhanced in hypergravity conditions

(Figure1). In terms of gender sub-analysis (supplementary material) it has been corroborated that males have a lower survival rate than females. Furthermore, it has been found that the male survival rate is less affected by gravitational changes (consistent with their smaller size and abdominal mass). Due to this fact, the contribution to the global increase in survival under hypergravity is mainly due to the male contribution for strain $\mathrm{L}$, whereas the decrease in survival observed in $\mathrm{M}$, and in a lesser extend for wt and S strains is mainly due to the female contribution.

The median survival for each strain with the $95 \%$ CI at $2 g$ and $1 g$ conditions is shown in Figure 2 . In $1 g$ control conditions, all strains show a similar median survival within the interval 20 to 24 days. However, when the flies are exposed to hypergravity, the median survival varies from $10 \pm 10$ days for the strain $\mathrm{M}$ to $31 \pm 3$ days for the strains B and L. Only the strain L presents a statistically meaningful median survival, when comparing $1 g / 2 g$.
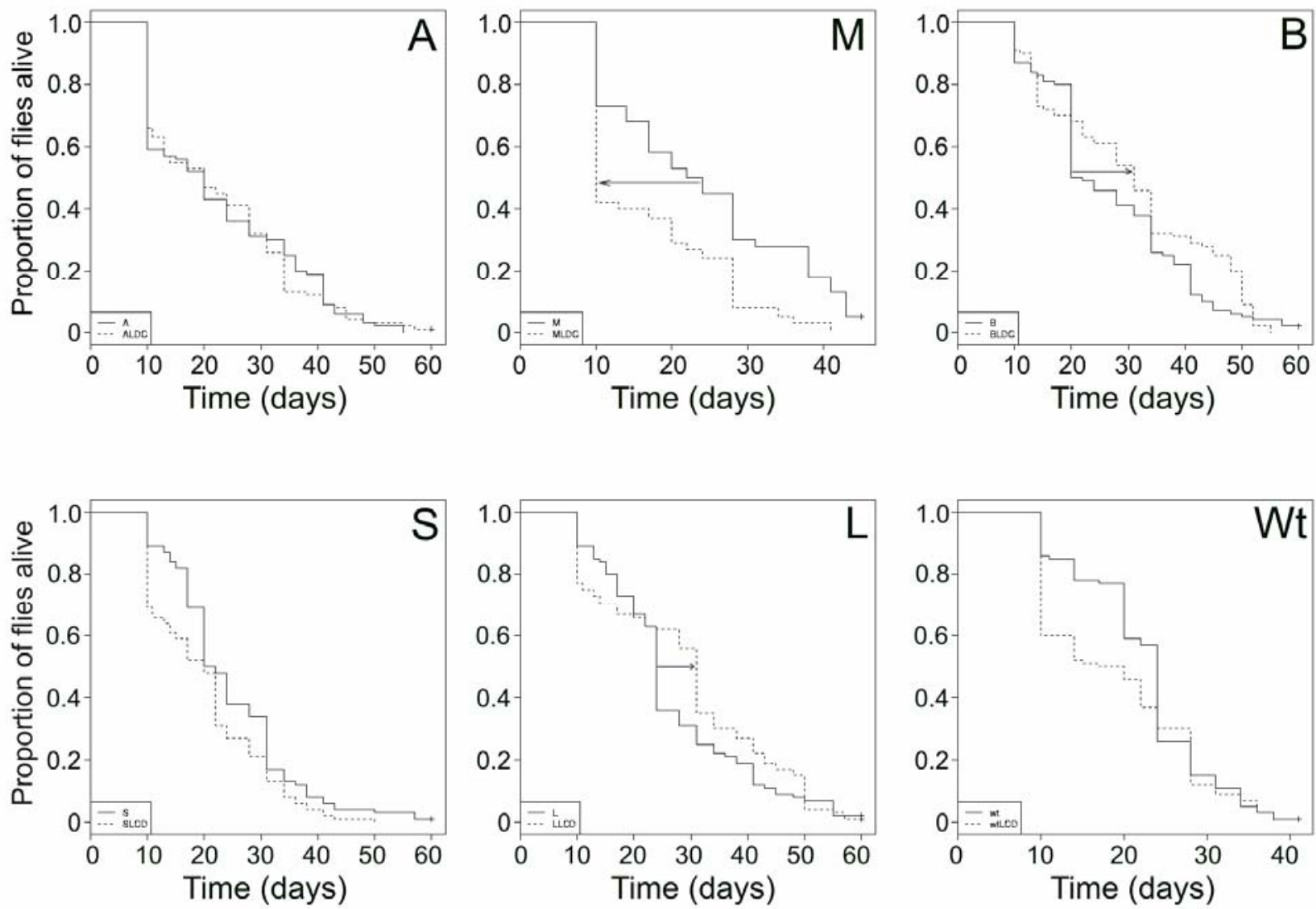

FIGURE 1. LOCF survival fit curves of the six Drosophila selected strains after a ten-day exposure to hypergravity conditions at $\mathbf{2 g}$. The survival rates of three altered-geotaxis responders (first row; negative geotaxis, A; neutral geotaxis, M; and positive geotaxis, B strain)) and two altered-ageing strains (second row; S, short life span and L, long life span) were compared with the wild-type strain (Wt). The dashed lines represent the survival of the flies exposed to $2 g$ for 10 days and brought back to $1 g$ ( $\mathrm{t} 0$ : start of the experiment, flies are placed in the LDC) and the solid lines show the survival of the corresponding controls at $1 \mathrm{~g}$. An arrow indicates the direction of the change on the median survival value, increasing in the case of the strains B (positive-geotaxis responders) and L (long-life) and decreasing for strain M (neutral-geotaxis responders). Additional data is provided as a supplementary material online. 


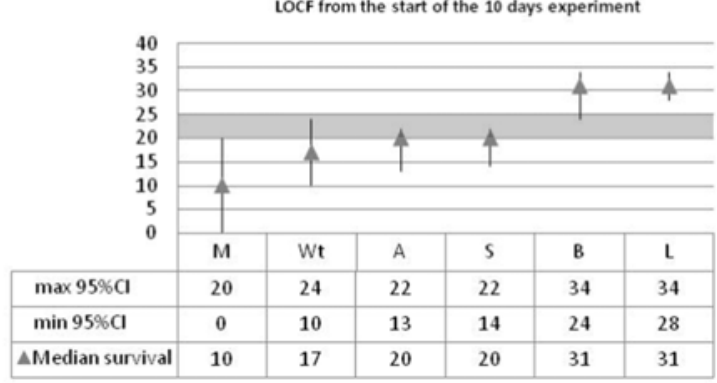

B)

\section{CONTROL Median survival (days)}

LoCF from the start of the 10 days experiment

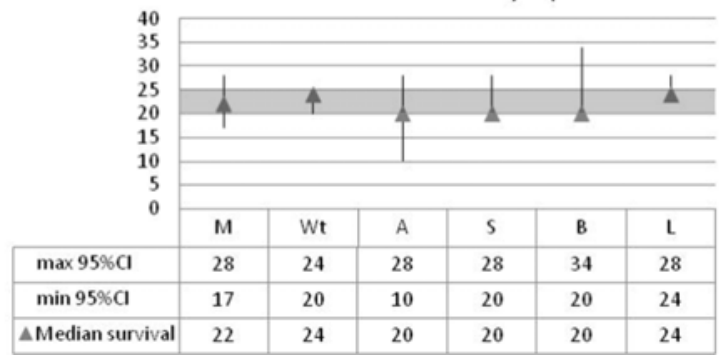

FIGURE 2. LOCF median survival of the six Drosophila strains including a 95\% CI under hypergravity (A) and at $\mathbf{1 g}$ control (B). Three alteredgeotaxis responders (negative geotaxis, $\mathrm{A}$; neutral geotaxis, M; and positive geotaxis, B) and two alteredageing strains (S, short life span and L, long life span) are the focus of the study; the wild-type strain (Wt) was included for comparison. All strains show a similar median survival in the interval 20 to 24 days in the control, with the exception of the reduced survival in the population $M$ (neutral-geotaxis responders) and the increased survival in the populations B (positive-geotaxis responders) and L (long-life).

\section{DISCUSSION}

Numerous experiments using Drosophila for space and gravitational research show an increased motility possibly due to its natural negativegeotactic behaviour that allow flies to use gravitational clues to escape from predators or look for food. In addition, an accelerated aging and decreased survival are broadly observed (Benguria et al. 1996; de Juan et al. 2007; Marco et al. 1996). In this study, we used both altered geotaxis (A, M, B) and survival (L and S) strains to prove the relation between the gravitational-driven behaviour and the survival. Hypergravity promotes an alteration on the flies' behaviour and motile pattern, leading to a significant decrease in the survival. The strain M (neutral-geotaxis responders) has been shown as the most sensitive to changes in gravity revealing a significant decrease in the survival under hypergravity conditions when compared to the controls at $1 \mathrm{~g}$. Furthermore, it was the only strain presenting an increased motility in $2 g$. On the contrary, the strains B (positive-geotaxis responders) and L (long-life) show a slight increase in the survival rates and no noticeable behavioural changes. It is speculated that the increment of the gravity force from $1 g$ to $2 g$ for the experiment, does not represent a great stress for flies and thus only the most sensitive strain (M) which does not respond to gravity in natural conditions (as if they lived in a continuous microgravity environment) is the one affected.

We hypothesise two major reasons to explain how the changes in the gravitational load has lead to a higher mortality rate in Drosophila flies exposed to altered gravity: 1) Behavioural or miss-orientation response, and 2) Energy consumption due to altered loading requirements. The first assumes that the changes in motility are due to a modification of the behaviour (and consequently alterations in behavioural gene expression) in an attempt to orientate and find the gravitational signal. This creates an environmental stress that will lead to an accelerated aging and a reduction of the survival. The second hypothesis regards the altered motility as an increment of the energy consumption, hence causing the acceleration of metabolic rates (and the corresponding modification on metabolic genes and/or enzymes expression), finally leading to a reduced survival.

Reviewing our previously published gene expression data (Herranz et al. 2010; Herranz et al. 2012b), we discovered that a small number of ontology groups, related with response to abiotic/biotic stress, together with the Osiris gene family, some reproduction-related genes and some odorant response proteins are consistently affected in adult individuals exposed to hypergravity conditions. All these genes have different or even unknown functions, but they share the common feature of being related with the behaviour of Drosophila. We also observed that altered gravity environments generated by magnetic fields cause a milder gene expression alteration than mechanical facilities in sessile samples (developing stages during metamorphosis) but greater effects when using adult flies as experimental samples. Moreover, gene expression is clearly affected by motility and behaviour in altered gravity environments. This was confirmed by adult flies, showing larger variations compared to sessile samples using magnetic levitation facilities (that best recreate microgravity environments), whereas the magnetic field itself does not affect neither motility nor fly orientation (Hill et al. 2012).

Gene expression, motility and aging in real or simulated altered gravity conditions have shown that behavioural responses are more relevant for motility than energy concepts. This is supported by the fact that different selected geotactic strains (A, $\mathrm{M}, \mathrm{B}$ ) orientate differently and have about the same median survival $(20,22$ and 20 days respectively) 
in normal gravity. This conclusion is valid for the first response to hypergravity in young animals, because in mature animals the response varies. In fact, L (long-life) mature imagoes (de Juan et al. 2007) do not show a highly accelerated aging process because they experience a minor increase or decrease in motility.

All these facts support the behavioural response hypothesis, proving it to be more consistent than the energy consumption hypothesis due to gravitational loading response in relation to altered motility (Figure 3). Nevertheless, energy concerns can be very important in less active animals (mature) or in sessile developmental stages (pupae) in which we have observed lower values for gene expression and motility activation. Similarly, accelerated aging is promoted by accelerated motility and also by changes in gene expression that are linked to behavioural responses to environmental stresses.

\section{CONCLUSIONS}

Motility patterns related with altered gravity are mainly driven by biological behaviour (not only by energy issues that should affect all strains equally) and aging is accelerated when adaptation to the environment is not optimal. Therefore, behavioural changes produced by a gravitational stress lead to an accelerated aging and a decreased survival in adult flies.

Populations of neutral-geotaxis responders (strain M) are the most sensitive to gravity alterations and show the highest impact on accelerated aging, together with an increased motility due to a poor adaptation to the new gravitational environment. In contrast, long-life and positive-geotaxis responders (strains L and B) seem better-adapted and present slightly higher survival rates.

\section{ACKLOWLEDGEMENTS}

The present research would not have been possible without the logistic support from the section TEC-MMG in ESTEC, especially Robert Lindner and Jutta Krause, as well as Dr. Lucas Sanchez, from the Laboratory at CIB. We want to acknowledge the mentorship of Prof. Roberto Marco, who was a pioneer of Space Biology Research in Spain, and we expect that this paper is worthy to be his memorial. H.C. Liedtke is acknowledged for his critical review and proofreading of the manuscript. PS \& RH were supported by a Spanish Science and Technology (Especializacion en Organismos Internacionales) / CDTI scholarship (ESA Spanish trainee program). RH was also supported by a CSIC JAE-Doc contract. This work was supported by Grants from the Spanish "Plan Nacional de Investigación Científica y Desarrollo Tecnológico" Ref. AYA2009-07792-E/ESP to RH, from the Dutch Organisation of Scientific research (NWO) \#MG-057 to JVL and by the access to the Ground Base Facilities: ESA SEGMGSPE_Ph1 Project to JvL, FJM and RH.

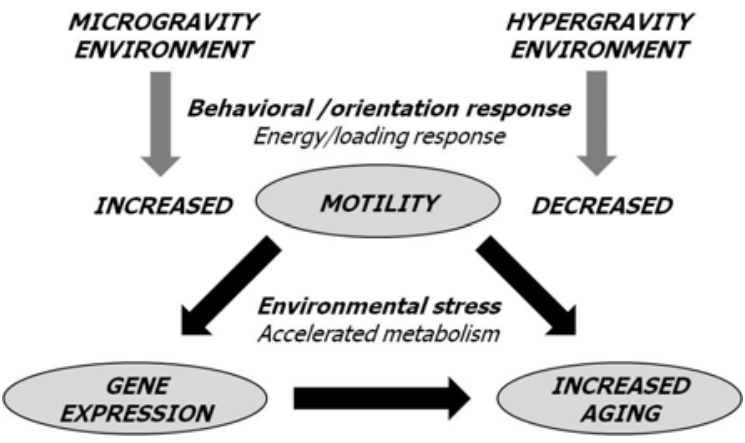

FIGURE 3. Relation among motility, gene expression and aging in altered gravity conditions. Behavioral responses and adaptation to environmental stress seem to have a larger contribution to accelerated aging than biophysical concepts like energy consumption and accelerated metabolism rates.

\section{REFERENCES}

Altman, D.G.: Practical Statistics for Medical Research, ed. Chapman \& Hall, London (1991)

Beaugnon, E., Tournier, R.: Levitation of organic materials. Nature 349, 470 (1991a)

Beaugnon, E., Tournier, R.: Levitation of water and organic substances in high static magnetic fields. J. Phys. III France 1, 1423-1428 (1991b)

Benguria, A., Grande, E., de Juan, E., Ugalde, C., Miquel, J., Garesse, R., Marco, R.: Microgravity effects on Drosophila melanogaster behavior and aging. Implications of the IML-2 experiment. J Biotechnol 47(2-3), 191-201 (1996)

de Juan, E., Benguría, A., Villa, A., Leandro, L.J., Herranz, R., Duque, P., Horn, E., Medina, F.J., Loon, J.v., Marco, R.: The "AGEING" Experiment in the Spanish Soyuz Mission to the International Space Station. Microgravity Sci Technol 19(3-4), 170-174 (2007)

Herranz, R., Anken, R., Boonstra, J., Braun, M., Christianen, P.C.M., Geest, M.d., Hauslage, J., Hilbig, R., Hill, R.J.A., Lebert, M., Medina, F.J., Vagt, N., Ullrich, O., Loon, J.J.W.A.v., Hemmersbach, R.: Ground-Based Facilities for Simulation of Microgravity, including Terminology and Organism-specific Recommendations for their use. Astrobiology 12 (12), in press (2012a)

Herranz, R., Benguria, A., Lavan, D.A., Lopez-Vidriero, I., Gasset, G., Medina, F.J., van Loon, J.J., Marco, R.: Spaceflight-related suboptimal conditions can accentuate the altered gravity response of Drosophila transcriptome. Mol Ecol 19, 4255-4264 (2010). doi:MEC4795 [pii]

10.1111/j.1365-294X.2010.04795.x

Herranz, R., Larkin, O.J., Dijkstra, C.E., Hill, R.J.A., Anthony, P., Davey, M.R., Eaves, L., van Loon, J.J.W.A., Medina, F.J., Marco, R.: Microgravity simulation by diamagnetic levitation: effects of a strong gradient magnetic field on the transcriptional profile of Drosophila melanogaster. BMC Genomics 13, 52 (2012b). doi:10.1186/1471-2164-13-52 
Herranz, R., Laván, D.A., Dijkstra, C., Larkin, O., Davey, M., Medina, F.J., van Loon, J.J.W.A., Marco, R., Schiller, P.: Drosophila Behaviour \& Gene expression in altered gravity conditions: Comparison between Space and ground facilities. Proc. of the 'Life in Space for Life on Earth Symposium', Angers, France, 22-27 June 2008, (ESA SP-663, December 2008). (2008)

Hill, R.J., Larkin, O.J., Dijkstra, C.E., Manzano, A.I., de Juan, E., Davey, M.R., Anthony, P., Eaves, L., Medina, F.J., Marco, R., Herranz, R.: Effect of magnetically simulated zero-gravity and enhanced gravity on the walk of the common fruitfly. J R Soc Interface 9 (72), 1438-1449 (2012). doi:10.1098/rsif.2011.0715

Lim, H.Y., Bodmer, R., Perrin, L.: Drosophila aging 2005/06. Exp Gerontol 41(12), 1213-1216 (2006). doi:S0531-5565(06)00330-5 [pii]

10.1016/j.exger.2006.10.013

Marco, R., Benguria, A., Sanchez, J., de Juan, E.: Effects of the space environment on Drosophila melanogaster development. Implications of the IML-2 experiment. J Biotechnol 47(2-3), 179-189 (1996)

Marco, R., Gonzalez-Jurado, J., Calleja, M., Garesse, R., Maroto, M., Ramirez, E., Holgado, M.C., de Juan, E., Miquel, J.: Microgravity effects on Drosophila melanogaster development and aging: comparative analysis of the results of the Fly experiment in the Biokosmos 9 biosatellite flight. Adv Space Res 12(1), 157-166 (1992)

Marco, R., Vernos, I., Gonzalez, J., Calleja, M.: Embryogenesis and aging of Drosophila melanogaster flown in the space shuttle. Preliminary analysis of experiment fly $15 \mathrm{E}$. Naturwissenschaften 73(7), 431-432 (1986)

Motulsky, H.: Intuitive Biostatistics. Chapter 6: Survival Curves. Oxford University Press. http://www.graphpad.com/www/book/survive.ht m (1995) 6th October 2011

R_Core_Team: R: a language and enviroment for statistical computing. http://www.R-project.org/ (2011).

Reiter, L.T., Potocki, L., Chien, S., Gribskov, M., Bier, E.: A systematic analysis of human diseaseassociated gene sequences in Drosophila melanogaster. Genome Res 11(6), 1114-1125 (2001). doi:10.1101/gr.169101

Serrano, P., van Loon, J.J., Manzano, A.I., Medina, F.J., Herranz, R.: Selection of Drosophila Altered Behaviour \& Aging strains for microgravity research. J Gravit Physiol, (2010). doi:http://hdl.handle.net/10261/39192

Valles, J.M., Jr., Guevorkian, K.: Low gravity on earth by magnetic levitation of biological material. $\mathrm{J}$ Gravit Physiol 9(1), P11-14 (2002)

van Loon, J.J.W.A.: Some history and use of the Random Positioning Machine, RPM, in gravity related research. Adv Space Res 39, 1161-1165 (2007)

van Loon, J.J.W.A., Krause, J., Cunha, H., Goncalves, J., Almeida, H., Schiller, P.: The large diameter centrifuge, LDC, for life and physical sciences and technology. Proc. of the 'Life in Space for Life on Earth Symposium', Angers, France 22-27 June 2008, (ESA SP-663, December 2008) (2008) 


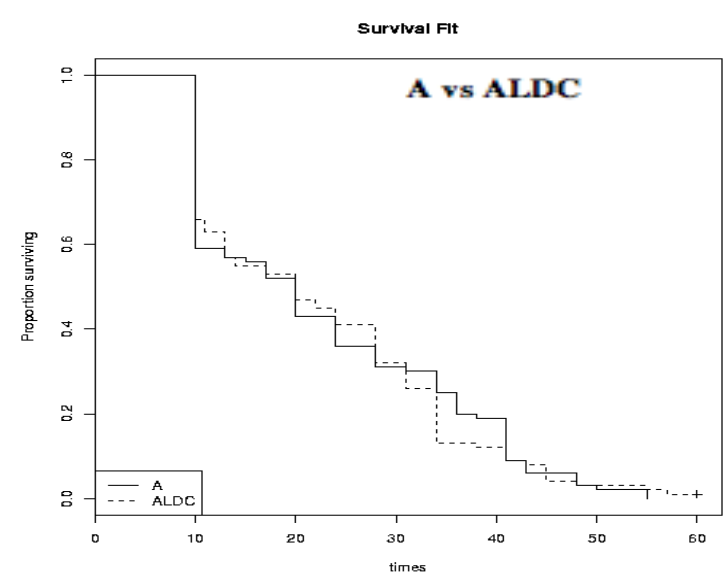

Chisq $=0$ on 1 degrees of freedom, $p=0.869$

Survival Fit

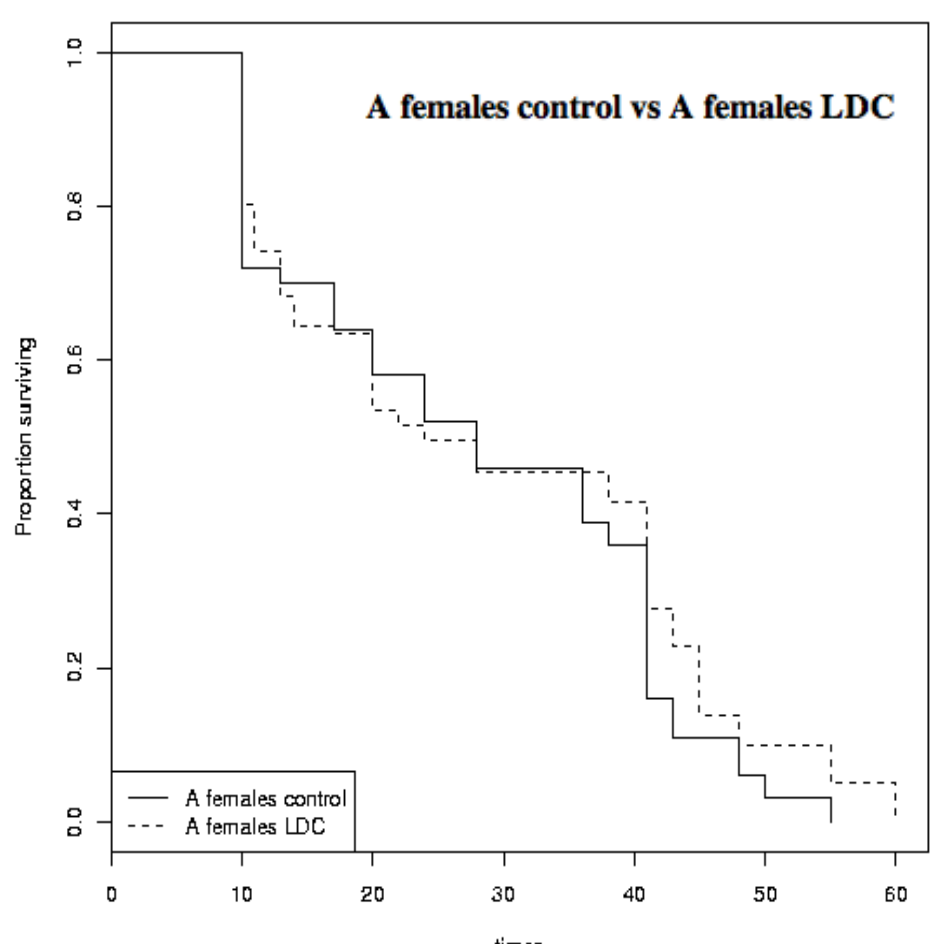

Chisq $=2.4$ on 1 degrees of freedom, $p=\mathbf{0 . 1 2}$
Fig SM1: To the left, the survival curves and results from the log rank test corresponding to the strain A (negative geotaxis, males and females together) in control (1g) and experimental conditions (exposure to $2 g$ over ten days).

Below, the survival curves and log rank tests corresponding to the female and male subpopulations, respectively. Note that in the case of the strain B, the survival in hypergravity slightly increases in the male subpopulation.

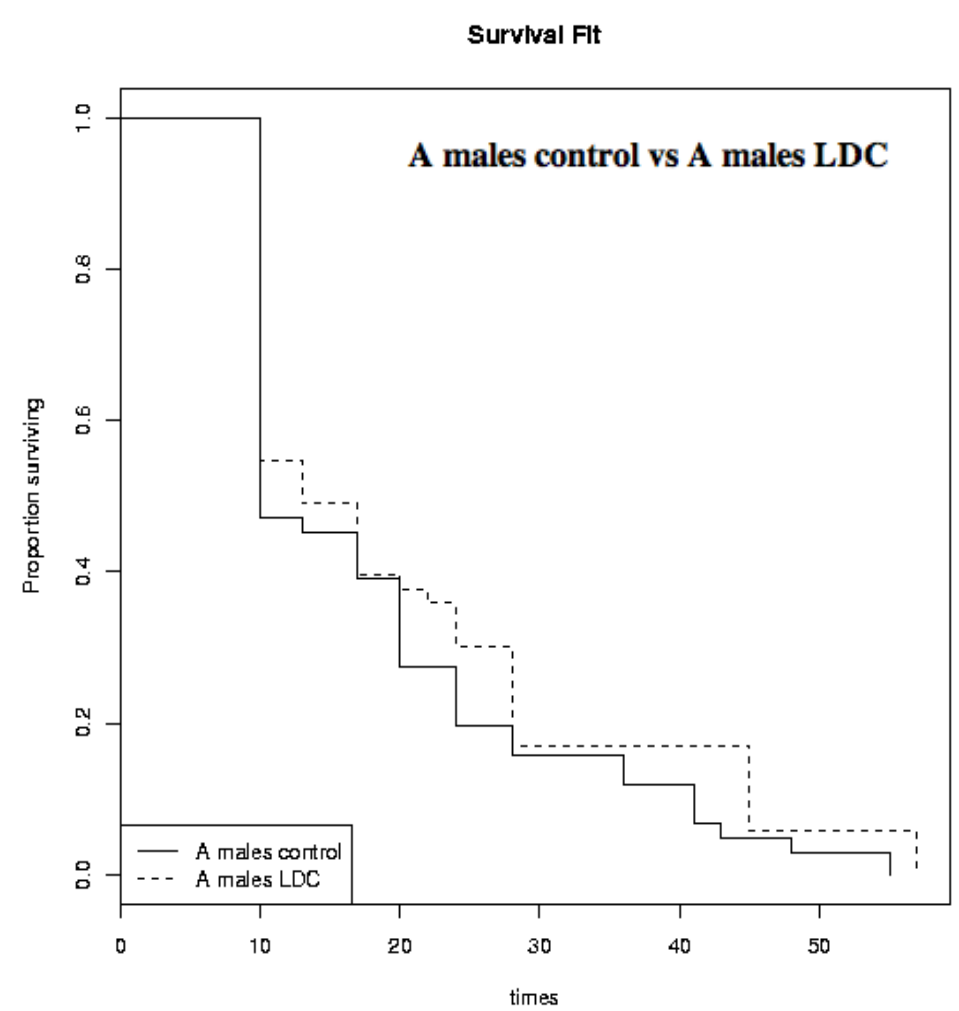

Chisq= 4.1 on 1 degrees of freedom, $p=0.0429$ 


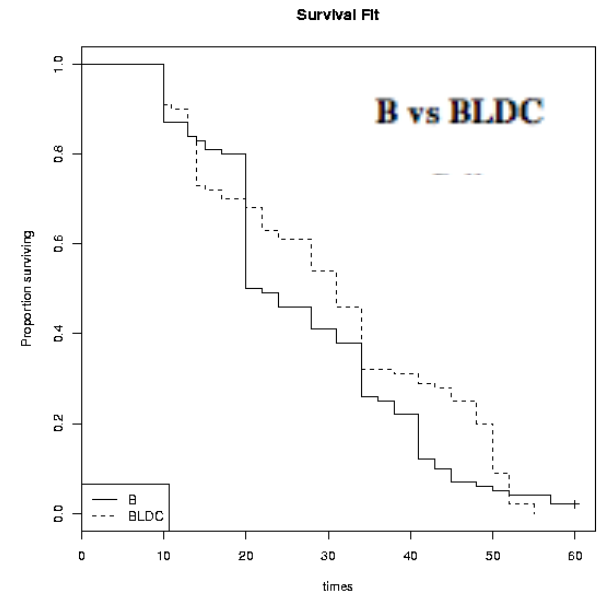

Chisq $=2.4$ on 1 degrees of freedom, $p=0.124$

Survival FIt

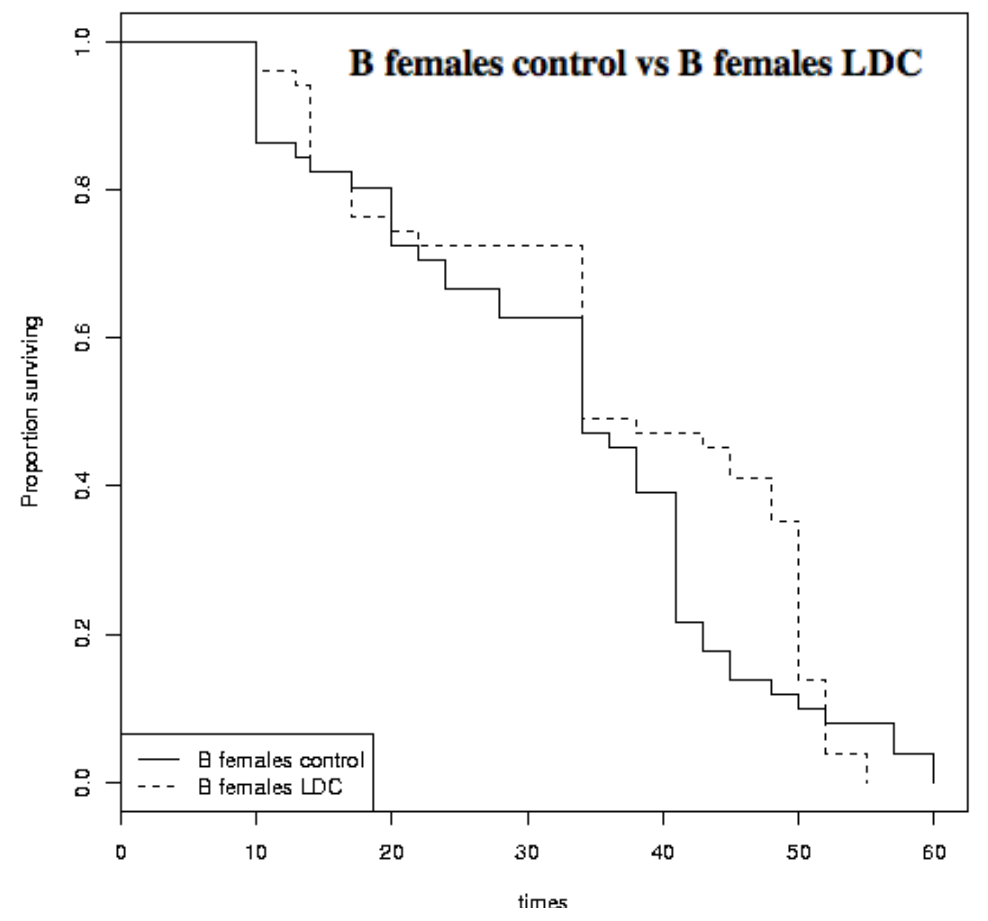

Chisq $=2.8$ on 1 degrees of freedom, $p=0.095$
Fig SM2: To the left, the survival curves and results from the log rank test corresponding to the strain B (positive geotaxis, males and females together) in control $(1 g)$ and experimental conditions (exposure to $2 g$ over ten days).

Below, the survival curves and log rank tests corresponding to the female and male subpopulations, respectively. Note that in the case of the strain B, the survival in hypergravity conditions slightly increases in the male population.

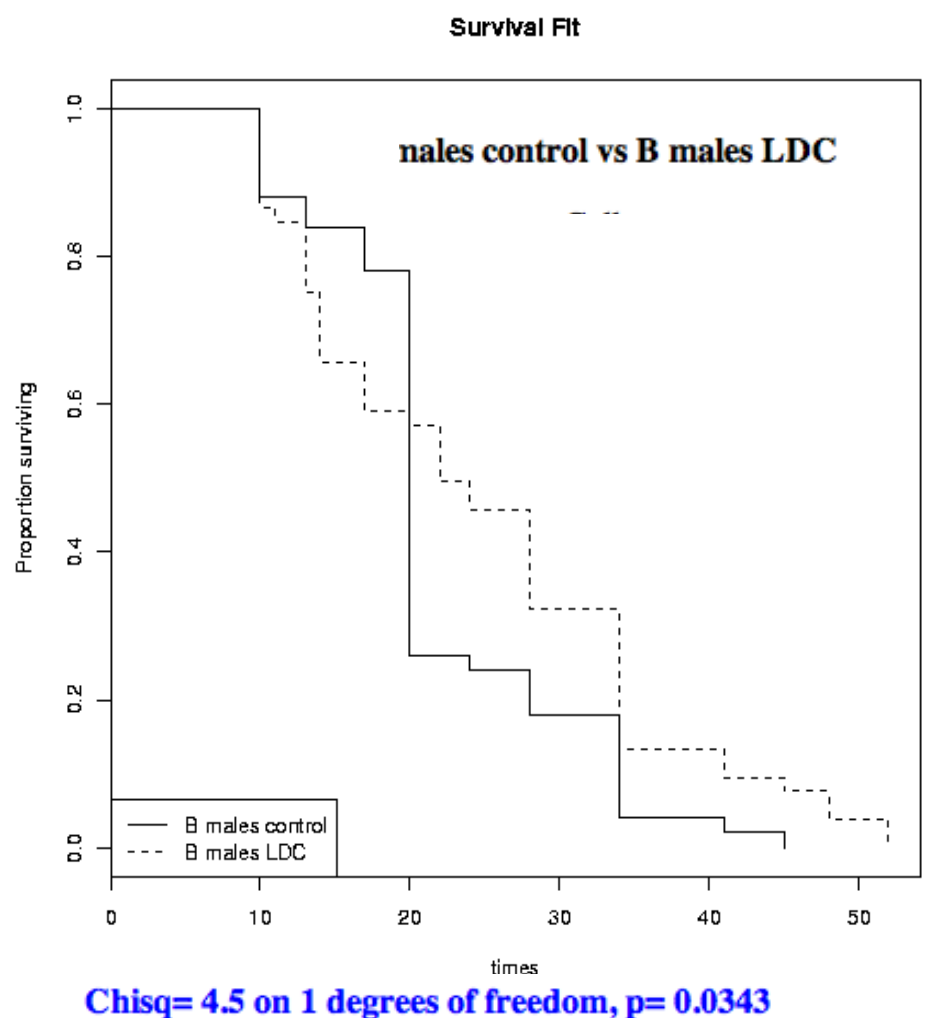

Chisq $=4.5$ on 1 degrees of freedom, $p=0.0343$ 


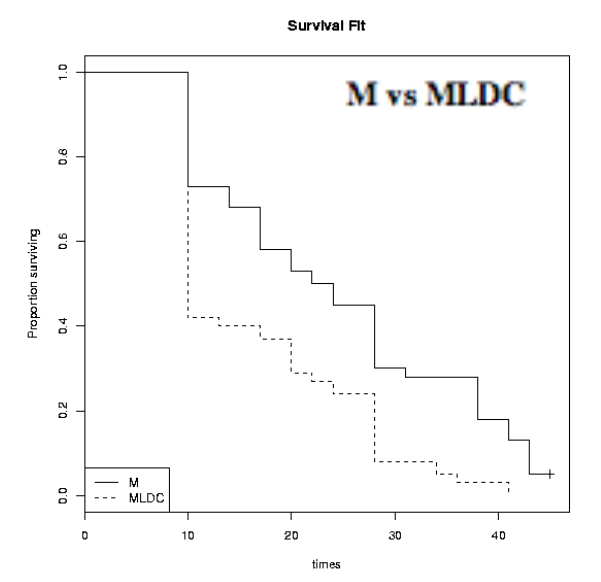

Chisq $=24.5$ on 1 degrees of freedom, $p=7.62 \mathrm{e}-07$

Survival FIt

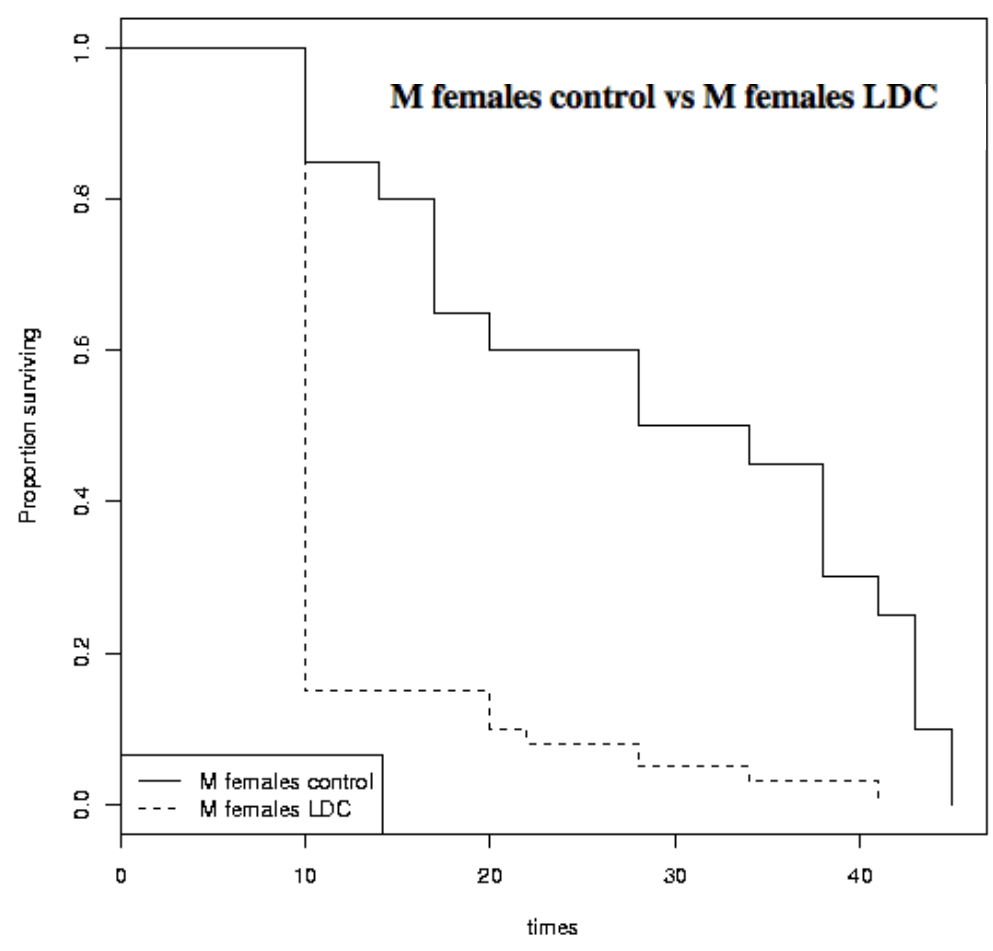

Chisq $=87$ on 1 degrees of freedom, $p=0$
Fig SM3: To the left, the survival curves and results from the $\log$ rank test corresponding to the strain M (neutral geotaxis, males and females together) in control $(1 g)$ and experimental conditions (exposure to $2 g$ over ten days).

Below, the survival curves and log rank tests corresponding to the female and male subpopulations, respectively. Note that in the case of the strain $\mathrm{M}$, the survival of the female individuals is the most affected, showing a noticeably reduced survival in hypergravity conditions.

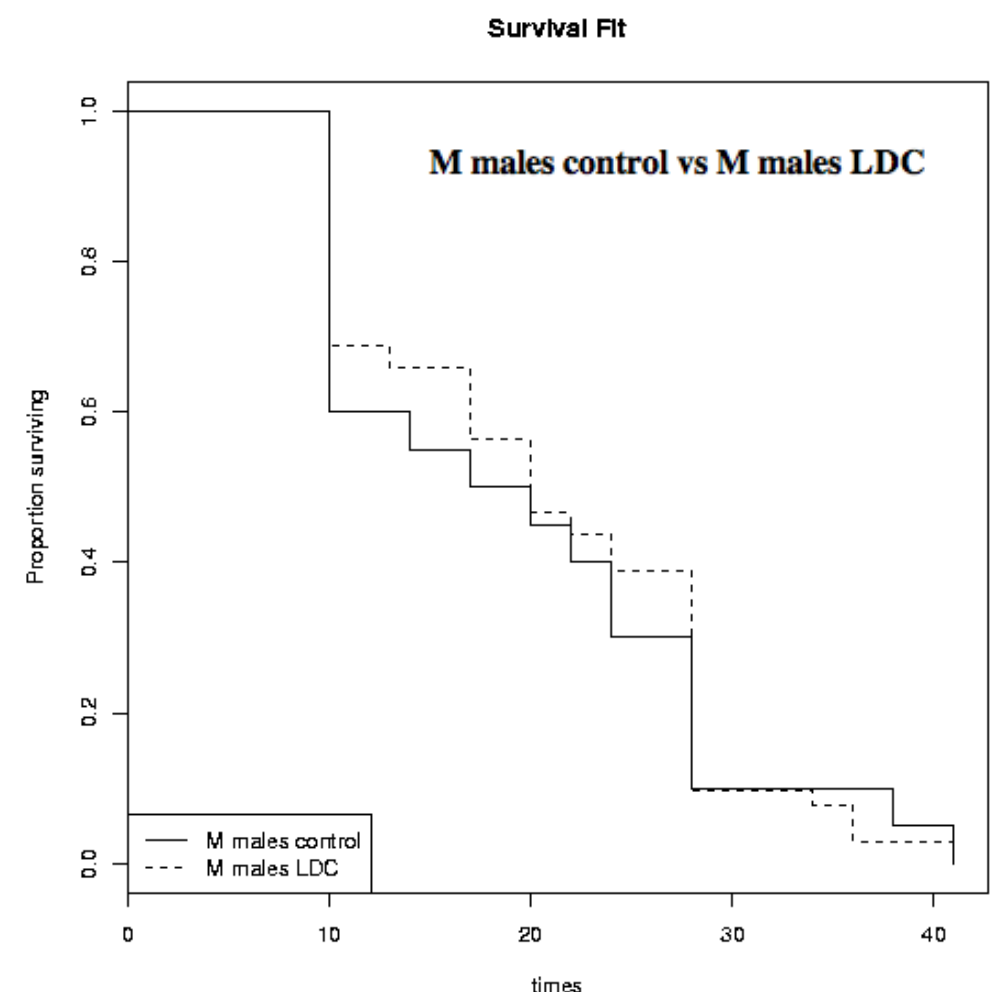

Chisq= 0.2 on 1 degrees of freedom, $p=0.694$ 


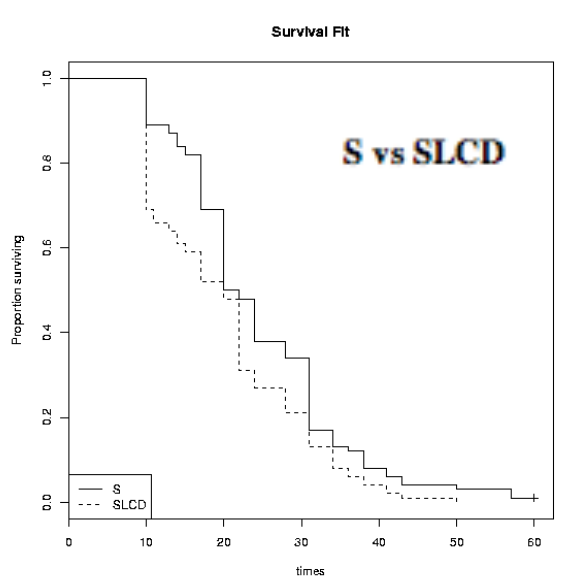

Chisq= 6.4 on 1 degrees of freedom, $p=0.0117$

Survival Fit

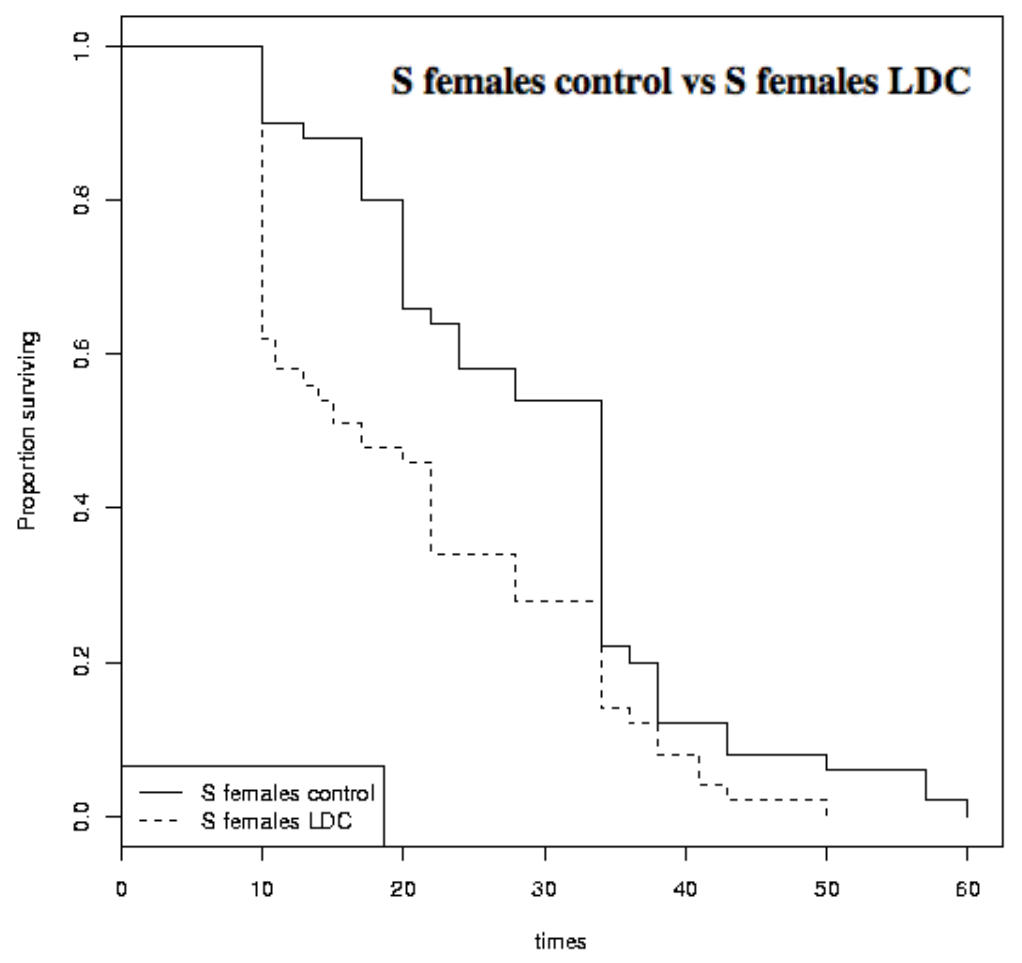

Chisq= 16.7 on 1 degrees of freedom, $p=4.41 e-05$
Fig SM4: To the left, the survival curves and results from the $\log$ rank test corresponding to the strain S (short life span, males and females together) in control $(1 g)$ and experimental conditions (exposure to $2 \mathrm{~g}$ over ten days).

Below, the survival curves and log rank tests corresponding to the female and male subpopulations, respectively. Note that in the case of the strain $\mathrm{S}$, the survival of the female subpopulation is considerably reduced in hypergravity conditions.

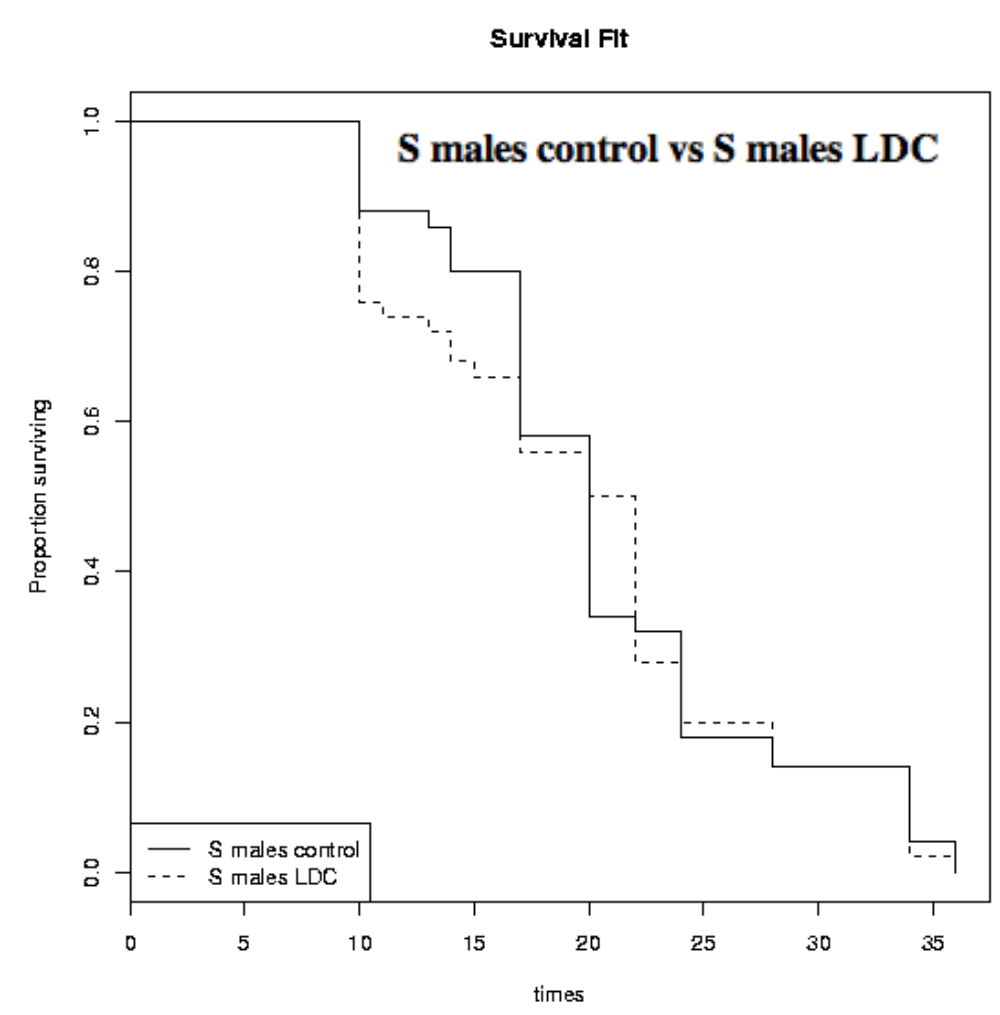

Chisq= 0.1 on 1 degrees of freedom, $p=0.765$ 


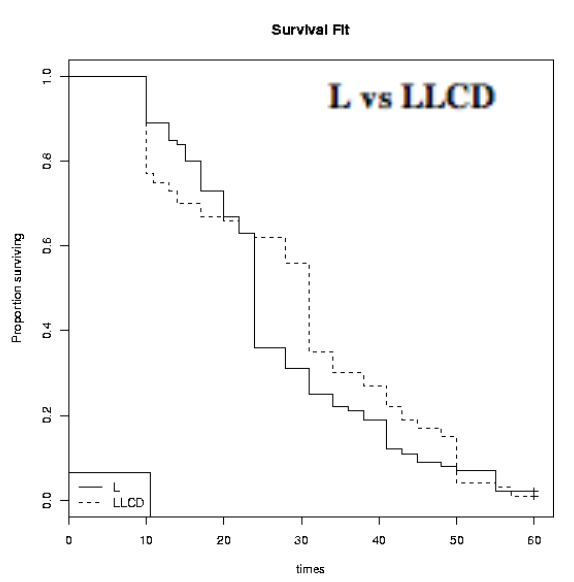

Chisq= 1.9 on 1 degrees of freedom, $p=0.163$

Survival Flt

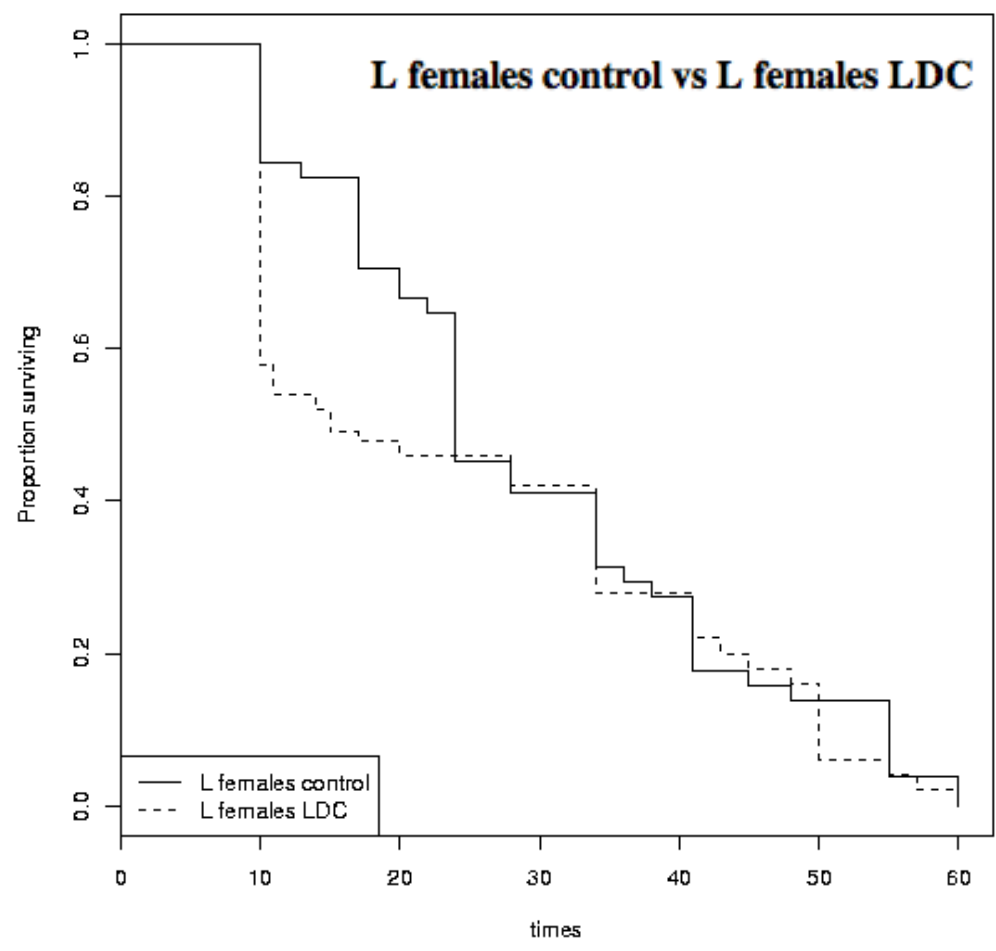

Chisq $=1.6$ on 1 degrees of freedom, $p=\mathbf{0 . 2 1 2}$
Fig SM5: To the left, the survival curves and results from the $\log$ rank test corresponding to the strain L (long life span, males and females together) in control (1g) and experimental conditions (exposure to $2 g$ over ten days).

Below, the survival curves and log rank tests corresponding to the female and male subpopulations, respectively. Note that in the case of the strain $\mathrm{L}$, the survival of the male individuals increases to a great extent.

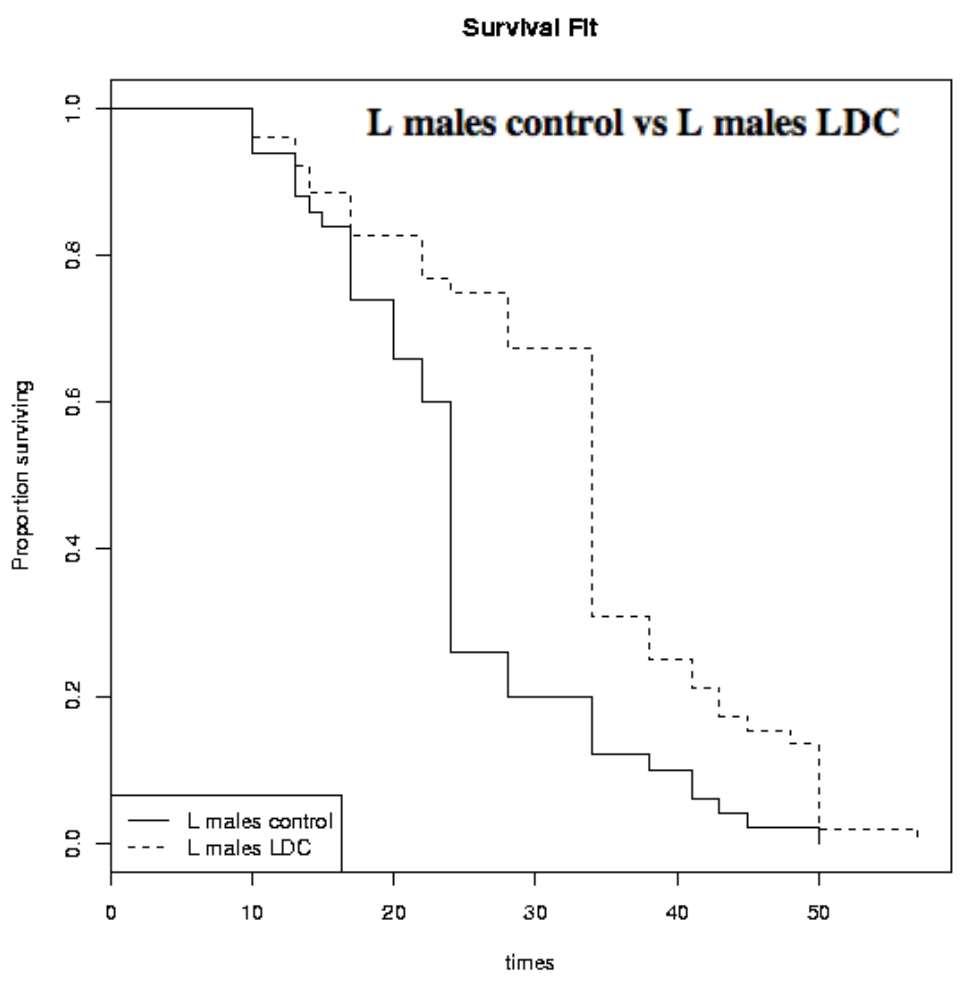

Chisq= 32.5 on 1 degrees of freedom, $p=1.19 \mathrm{e}-08$ 


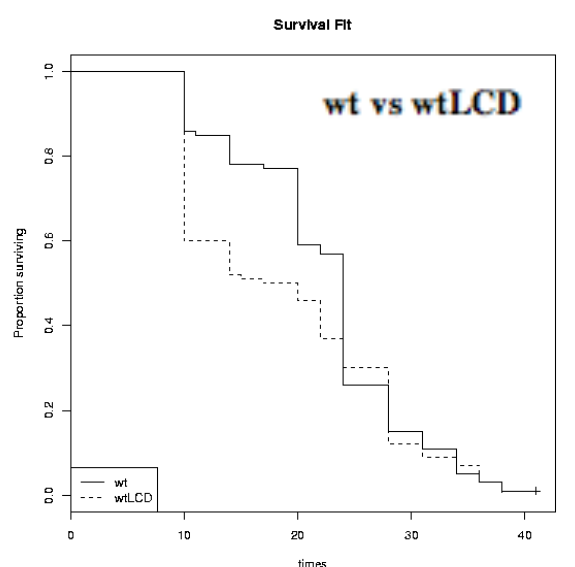

Chisq $=\mathbf{2 . 2}$ on 1 degrees of freedom, $p=0.142$

Survival Fit

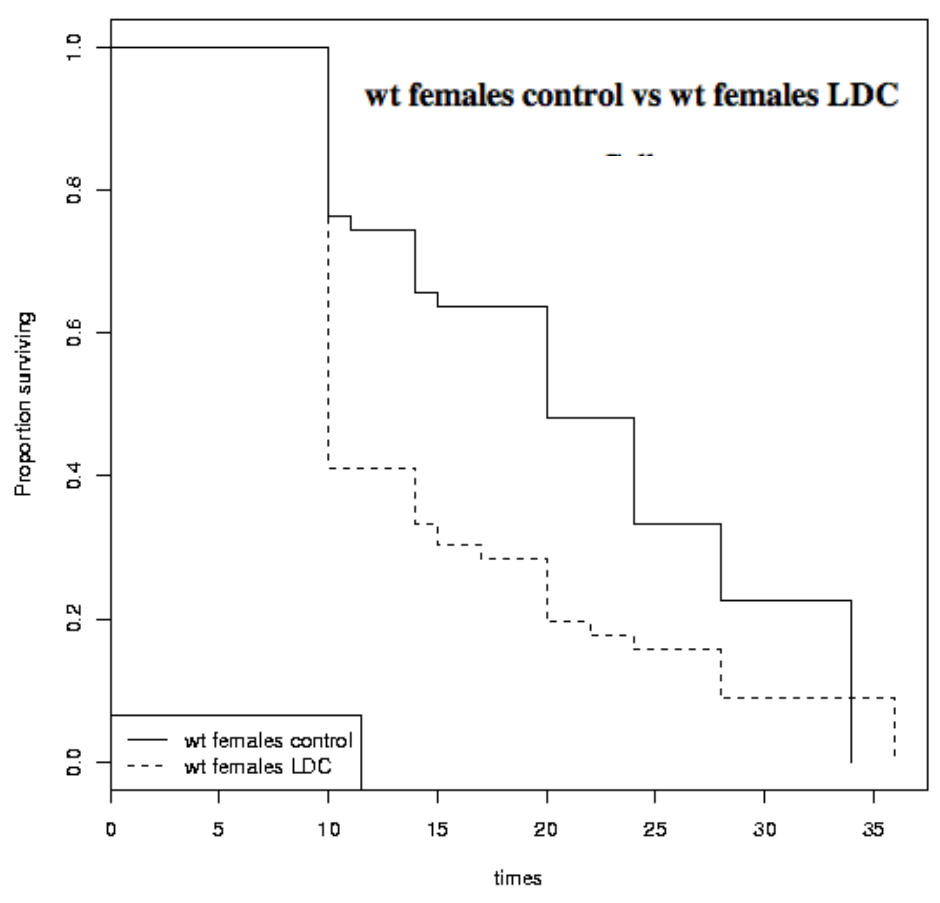

Chisq= 9.9 on 1 degrees of freedom, $p=\mathbf{0 . 0 0 1 6 3}$
Fig SM6: To the left, the survival curves and results from the log rank test corresponding to the Drosophila wild-type, wt (males and females together) in control $(1 g)$ and experimental conditions (exposure to $2 g$ over ten days).

Below, the survival curves and log rank tests corresponding to the female and male subpopulations, respectively. Note that in the case of the wt flies, the survival of the female individuals is the most affected, showing a decrease in hypergravity conditions.

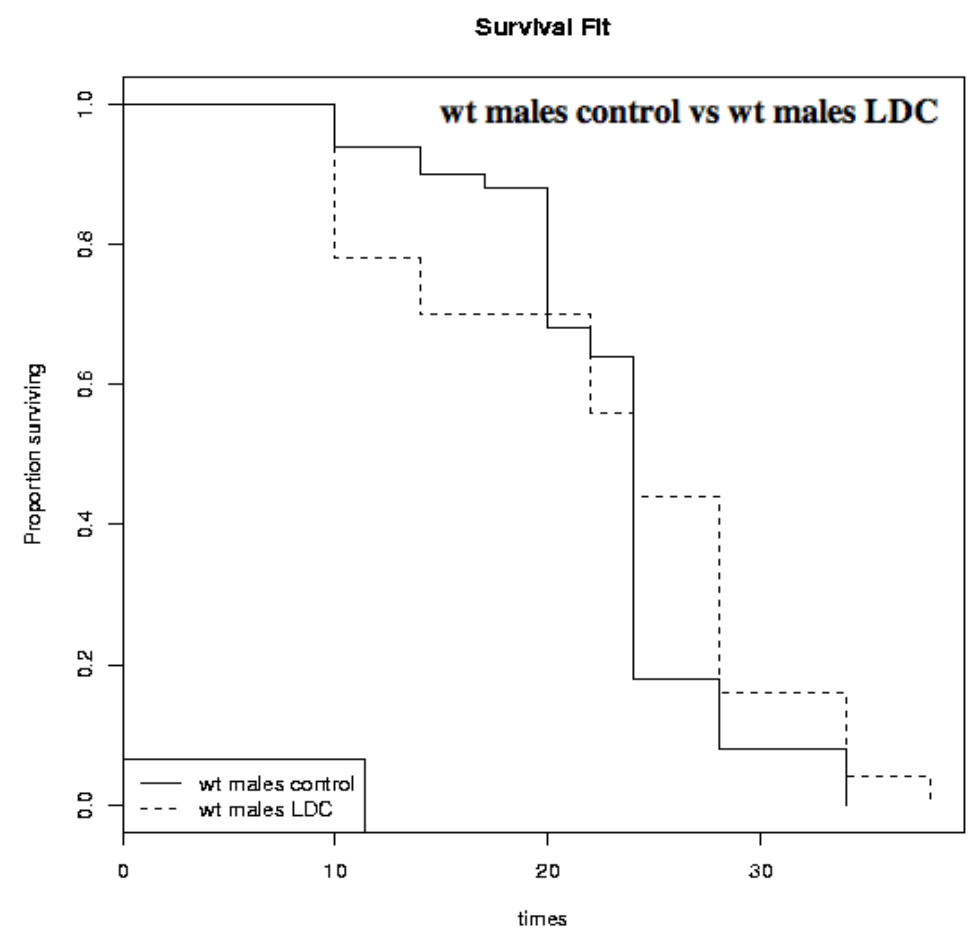

Chisq $=3.2$ on 1 degrees of freedom, $p=0.0723$ 